\title{
Influence of iron on sperm motility and selected oxidative stress parameters in fertile males - a pilot study
}

\author{
Aleksandra Kasperczyk', Michał Dobrakowski', Zenon P. Czuba², Lucyna Kapka-Skrzypczak ${ }^{3,4}$, \\ Sławomir Kasperczyk ${ }^{1}$ \\ ${ }^{1}$ Medical University of Silesia in Katowice, Department of Biochemistry, Zabrze, Poland \\ ${ }^{2}$ Medical University of Silesia in Katowice, Department of Microbiology and Immunology, Zabrze, Poland \\ ${ }^{3}$ Department of Molecular Biology and Translational Research, Institute of Rural Health, Lublin, Poland \\ ${ }^{4}$ Department of Medical Biology and Translational Research, Faculty of Medicine, University of Information Technology \\ and Management, Rzeszów, Poland
}

Kasperczyk A, Dobrakowski M, Czuba ZP, Kapka-Skrzypczak L, Kasperczyk S. Influence of iron on sperm motility and selected oxidative stress parameters in fertile males - a pilot study. Ann Agric Environ Med. 2016; 23(2): 292-296. doi: 10.5604/12321966.1203893

\section{Abstract}

Introduction. The presented study was designed to investigate the associations between iron level in seminal plasma and levels of the parameters of oxidative stress and antioxidant defence system and selected cytokines in the seminal plasma of fertile males.

Materials and methods. The study population consisted of 65 fertile male volunteers from the southern region of Poland. Based on the medians of the levels of iron in seminal plasma, the study subjects were divided into 2 groups: with low and high levels of iron in seminal plasma (Fe-L and Fe-H).

Results. The percentage of unprogressively motile sperm cells after 1 hour was significantly higher in the Fe- $\mathrm{H}$ group, compared to the Fe-L group. The activities of SOD and Mn-SOD and the level of MDA were significantly lower in the Fe-H group, compared to the Fe-L group. However, the value of TOC and OSI were significantly higher in the Fe-H group, compared to the Fe-L group. Median of IL-1 $\beta$ was significantly higher in the Fe-H group, compared to the Fe-L group, whereas medians of IL-2, IL-5, and GM-CSF were significantly lower in the Fe-H group.

Conclusion. In fertile males, iron may negatively affect sperm motility and elevate oxidative stress intensity. Iron additionally modulates the levels of several cytokines in human semen.

\section{Key words}

iron, semen, oxidative stress, cytokines, fertility

\section{INTRODUCTION}

The role of trace elements in sperm may have significant influence upon male infertility. Iron (Fe) is among these elements which participates in oxygenation and reduction processes, entering into the composition of many enzymes and metalloprotein compounds. Although Fe and its compounds are not essentially toxic for human and animals, disturbances can appear due to prolonged intake of high doses of Fe or dysfunction of the regulatory mechanisms of Fe metabolism [1].

Maturating germ cells are compartmentalized by Sertoli cell tight junctions. It has been proposed that the Sertoli cells bind and internalize diferric transferrin from the blood at their basal pole, remove iron from the basal compartment, and provide it to the meiotic spermatocytes and differentiating spermatids located in the adluminal compartment of the seminiferous epithelium. In seminiferous tubules, iron is bound to a testicular transferrin secreted by Sertoli cells, and is internalized by the maturating germ cells through transferrin receptors [2]. The latest reports indicate that iron metabolism in mitochondria is crucial for energy production and spermatogenesis. Therefore, the transport of iron from cytoplasm to mitochondria, facilitated by the mitochondrial carrier proteins, is also under investigation [3].

Address for correspondence: Aleksandra Kasperczyk, Medical University of Silesia in Katowice, Department of Biochemistry, Jordana 19, 41-808 Zabrze, Poland,

E-mail: olakasp@poczta.onet.pl

Received: 01 May 2014; accepted: 07 July 2014
It has been reported that $\mathrm{Fe}$ can cause an increase in sperm damage [4]. The toxicity of Fe could result from the Fenton and Haber-Weiss reactions resulting in the formation of hydroxyl free radicals from hydrogen peroxide and superoxide ion radicals. The prime targets of these radicals are polyunsaturated fatty acids of the cell membranes. Damage to these fatty acids results in impairment of the cell structure and function. Because testicular tissue is susceptible to attack by the reactive oxygen species, Fe-induced oxidative stress may induce lipid peroxidation, which has been shown to lead to the deterioration of sperm morphology and motility $[5,6]$. On the other hand, spermatozoa and seminal plasma possess anti-oxidant systems capable of counteracting the harmful effects of ROS [7]. The seminal fluid normally contains high amounts of non-enzymatic antioxidants, such as uric acid, thiol groups, glutathione, spermin, and vitamin C, and enzymatic antioxidants, such as superoxide dismutase (SOD) or catalase (CAT) [8]. Besides, the normal sperm function is controlled by the neuroendocrine-immune network integrated by cytokines [9]. However, the associations between Fe and cytokine levels in semen are poorly recognized.

In light of the above-mentioned information, the role of Fe in spermatogenesis and sperm function is indispensable; however, Fe ions may have also toxic effects on sperm. Therefore, the presented study was designed to explore the possible effects of Fe toxicity on the sperm quality of fertile males. For this purpose, the associations between Fe levels and levels of oxidative stress (OS) markers, such 
as malondialdehyde (MDA), total oxidant status (TOS), and oxidative stress index (OSI) in seminal plasma, were investigated. Total antioxidant capacity (TAC) value and levels of the protein sulfhydryl groups (PSH), total activity of superoxide dismutase (SOD) and activities of its izoenzymes, such as Mn-SOD and CuZn-SOD in seminal plasma, were also measured. Additionally, MDA concentration and SOD activity were determined in spermatozoa. Simultaneously, the relationship was determined between Fe level and the plasma levels of cytokines: pro-inflammatory cytokines (IL-1 $\beta$, IL-6, IL-8, IL-12), cytokines related to the Th type 1 response (IL-2, INF- $\gamma$ ), cytokines related to the Th type 2 response (IL-4 and IL-5), and colony stimulating factors (G-CSF and GM-CSF).

\section{MATERIALS AND METHOD}

Study population. Before sarting the trial, the authors obtained the consent of the Institutional Ethics Committee (Permission No. KNW/0022/KB1/13/09). The study population consisted of 65 healthy, non-smoking, fertile men from the southern region of Poland. Patients were drug tested, and no drug consumption (including antioxidant medications) was reported at the time of the study. All the participants had normal semen specimens according to the WHO standards [10]. Based on the median of the values of iron concentration in seminal plasma $(\mathrm{FeS}=1.80 \mu \mathrm{g} / \mathrm{dl})$, the subjects were divided into 2 groups:

1) with a low iron level (LE-Fe) - iron concentration in seminal plasma between 0.10 and $3.50 \mu \mathrm{g} / \mathrm{dl} ; \mathrm{n}=33$;

2) with a high iron level (HE-Fe) - iron concentration in seminal plasma between 3.51 and $21.0 \mu \mathrm{g} / \mathrm{dl} ; \mathrm{n}=32$.

Samples collection. Semen was collected on the same day, in the morning before the first meal. Semen samples $(2-6 \mathrm{ml})$ were collected by masturbation, at home or at laboratory research facilities, after at least 3 days of sexual abstinence (number of days elapsed since last ejaculation was recorded for each volunteer).

Semen analysis. All the semen specimens were analyzed according to WHO standards [10], including assessment of the seminal volume, sperm cell density, total sperm cell count, motility, and supravital eosin staining (for percentage of live spermatozoa). Sperm morphology was examined after Papanicolaou staining. The semen samples $(1.5 \mathrm{ml})$ after liquefaction were centrifuged at $6,000 \mathrm{~g}$ for $10 \mathrm{~min}$. to separate the spermatozoa from the seminal plasma. The seminal plasma was transferred to fresh tubes and stored at $-75^{\circ} \mathrm{C}$ until required for the biochemical and lead analyses. Additionally, a $10 \%$ spermatozoa lysate in bi-distilled water was prepared.

Determination of iron. Iron concentration in seminal plasma was determined photometrically. In $\mathrm{pH}<7$ iron is released from transferrin. Ascorbate reduces $\mathrm{Fe}^{3+}$ ions to $\mathrm{Fe}^{2+}$, which then react with ferrozine to form a coloured complex. The colour intensity is directly proportional to the iron concentration, expressed in $\mu \mathrm{g} / \mathrm{dl}$.

Determination of Protein Sulfhydryl Groups (PSH). PSH concentration in seminal plasma was determined as described by Koster et al. [11] using DTNB, which undergoes reduction by compounds containing sulfhydryl groups, yielding the yellow anion derivative, 5-thio-2-nitrobenzoate, which absorbs at a wavelength of $412 \mathrm{~nm}$ using an automated Perkin Elmer analyzer. The results were shown in $\mu \mathrm{mol} / \mathrm{g}$ protein.

Determination of Superoxide Dismutase (SOD) activity. The method of Oyanagui [12] was used to measure the activity of SOD in seminal plasma and in spermatozoa. In this method, xanthine oxidase produces superoxide anions which react with hydroxylamine, forming nitric ions. This ions react with naphthalene diamine and sulfanilic acid generating a coloured product. The concentration of this product is proportional to the amount of produced superoxide anions and negatively proportional to the activity of SOD. Absorbance was measured using an automated Perkin Elmer analyzer at the wavelength of $550 \mathrm{~nm}$. The enzymatic activity of SOD was expressed in nitric units. The isoenzymes of SOD, such as Mn-SOD and CuZn-SOD, were also indicated, using KCN as the inhibitor of the CuZn-SOD activity. The activity of SOD is equal to 1 nitric unit (NU) when it inhibits nitric ion production by $50 \%$. Activities of $\mathrm{SOD}$ in seminal plasma were expressed in NU/mg protein and in NU/100 $\mu \mathrm{l}$ packed spermatozoa.

Determination of Malondialdehyde (MDA). MDA, a product of lipid peroxidation, was measured fluorometrically as a 2-thiobarbituric acid-reactive substance (TBARS) in seminal plasma and lysate of spermatozoa, according to Ohkawa et al. [13] with modifications. Samples were mixed with $8.1 \%$ sodium dodecyl sulphate, $20 \%$ acetic acid and $0.8 \%$ 2-thiobarbituric acid. After vortexing, samples were incubated for 1 hour in $95^{\circ} \mathrm{C}$, and butanol-pyridine 15:1 (v/v) was added. The mixture was shaken for 10 minutes and then centrifuged. The butanol-pyridine layer was measured fluorometrically at $552 \mathrm{~nm}$ and $515 \mathrm{~nm}$ excitation (Perkin Elmer, USA). TBARS values are expressed as malondialdehyde (MDA) equivalents. Tetraethoxypropane was used as the standard. Concentrations are given in $\mu \mathrm{mol} / \mathrm{l}$ plasma and $\mu \mathrm{mol} / \mathrm{dl}$ in packed spermatozoa.

Determination of Total Oxidation Status (TOS). Total oxidant status was measured in seminal plasma according to Erel [14]. The assay is based on the oxidation of ferrous ions to ferric ions in the presence of various oxidant species in acidic medium. The colour change of xylenol orange by the ferric ions is measured as the change in absorbance at $560 \mathrm{~nm}$. This method was conducted in an automated analyzer (Perkin Elmer) calibrated with hydrogen peroxide. Data are shown in $\mu \mathrm{mol} / \mathrm{l}$.

Determination of Total Antioxidant Capacity (TAC). Total antioxidant capacity was measured according to Erel [15]. In this colourimetric method, radicals are generated and the antioxidant activity of seminal plasma reduces radical formation. The change in colour of $\mathrm{ABTS}^{+}$ions (2,2'-azinobis(3-ethylbenzothiazoline-6-sulfonate) is measured as the change in absorbance at $660 \mathrm{~nm}$. This method was conducted in an automated Perkin Elmer analyzer calibrated with Trolox. Data are shown in mmol/l.

Oxidative Stress Index (OSI). The percentage ratio of TOS to TAC was used as the OSI. 
Determination of cytokines. IL-1 $\beta$, IL-2, IL-4, IL-5, IL-6, IL-8, IL-12, INF- $\gamma$, G-CSF, GM-CSF were detected in seminal plasma using the Bio-Plex 200 System (Bio-Rad Laboratories Inc., USA), which is based on 3 core elements. The first core element is a technology that uses fluorescently-dyed magnetic microspheres (beads), each with a distinct colour code to permit discrimination of individual tests within a multiplex suspension, and allows the simultaneous detection of diverse analyte molecules in a single well of a 96-well microplate. Moreover, the magnetic beads allow for magnetic separation during the washing steps. The second core element is a dedicated flow cytometer with 2 lasers (a $532 \mathrm{~nm} \mathrm{Nd-Yag}$ laser used to excite phycoerythrin in the assay, and a $635 \mathrm{~nm}$ solid state laser used to excite the dyes inside the beads to determine their 'colour' or 'region') and associated optics to measure the different molecules bound to the surface of the beads. The third core element is a high-speed digital signal processor that efficiently manages the fluorescent output.

The principle of these bead-based assays is similar to that for capturing sandwich immunoassays. The samples and standards were incubated with the coupled beads (antibodies directed against the desired cytokines were covalently coupled to internally-dyed beads) in the wells of 96-well plates, and washed. Next, the biotinylated detection antibodies specific for different cytokine epitopes were added. After incubation and washing, streptavidin (phycoerythrin solution) was added to bind biotinylated detection antibodies on the beads. Next, the suspensions of washed beads were analyzed using the Bio-Plex System. Software data presented as both median fluorescence intensity and concentration ( $\mathrm{pg} / \mathrm{ml})$.

Statistical analysis. A database was created in the MS Excel 2007. Statistical analysis was performed using Statistica 10.0 PL software. Statistical methods included mean and standard deviation (SD) for normal distribution, and median and interquartile range (IQR) for abnormal distribution. ShapiroWilk's test was used to verify normality and Levene's test to verify homogeneity of variances. Statistical comparisons between groups were made by a t-test, t-test with a separate variance, or Mann-Whitney U test (non-parametric test). Spearman's coefficient $R$ for non-parametric correlation was calculated. A value of $\mathrm{p}<0.05$ was considered significant.

\section{RESULTS}

Mean age in the Fe-L and $\mathrm{Fe}-\mathrm{H}$ groups did not differ significantly. There was a strong tendency towards lower sperm volume by $19 \%$ in the Fe-H group, compared with the Fe-L group. Similarly, an analogous tendency was observed for the percentage of progressively motile sperm cells after 1 hour, which was lower in the $\mathrm{Fe}-\mathrm{H}$ group by $15 \%$. However, the percentage of unprogressively motile sperm cells after 1 hour was significantly higher in the Fe-H group, compared to the Fe-L group, by $34 \%$. There was a strong tendency towards a higher percentage of low progressively motile sperm cells after 1 hour by $21 \%$. Sperm pH, count, morphology and other parameters characterizing motility did not differ between the analyzed groups (Table 1).

The activities of SOD and Mn-SOD were significantly lower in the Fe-H group, compared to the Fe-L group by $64 \%$ and $63 \%$, respectively. Similarly, MDA concentration was significantly lower by $25 \%$. However, the values of TOC and
Table 1. Semen parameters and iron concentration in the study population

\begin{tabular}{|c|c|c|c|c|c|c|}
\hline & $\begin{array}{l}\text { Fe-L } \\
\text { group } \\
\mathrm{n}=33\end{array}$ & $\begin{array}{c}\mathrm{Fe}-\mathrm{H} \\
\text { group } \\
\mathrm{n}=32\end{array}$ & & & $p$ value & $\begin{array}{l}\text { relative } \\
\text { change }\end{array}$ \\
\hline & Mean & SD & Mean & SD & & \\
\hline Age & 32.8 & 5.28 & 32.3 & 6.24 & 0.732 & $-2 \%$ \\
\hline Volume & 4.41 & 2.24 & 3.55 & 1.00 & 0.052 & $-19 \%$ \\
\hline $\mathrm{pH}$ & 7.58 & 0.07 & 7.56 & 0.07 & 0.258 & $-0,3 \%$ \\
\hline $\begin{array}{l}\text { Sperm cells count in } 1 \mathrm{ml} \\
(\mathrm{mln} / \mathrm{ml})\end{array}$ & 68.5 & 50.6 & 84.2 & 57.5 & 0.248 & $23 \%$ \\
\hline $\begin{array}{l}\text { Total sperm cells count } \\
(\mathrm{mln})\end{array}$ & 276 & 197 & 283 & 180 & 0.870 & $3 \%$ \\
\hline $\begin{array}{l}\text { Motile sperm cells after } 1 \\
\text { hour (\%) }\end{array}$ & 59.0 & 10.93 & 58.0 & 10.16 & 0.695 & $-2 \%$ \\
\hline $\begin{array}{l}\text { Progressively motile sperm } \\
\text { cells after } 1 \text { hour (\%) }\end{array}$ & 29.0 & 11.6 & 24.6 & 8.53 & 0.084 & $-15 \%$ \\
\hline $\begin{array}{l}\text { Low progressively motile } \\
\text { sperm cells after } 1 \text { hour (\%) }\end{array}$ & 16.1 & 5.95 & 19.5 & 7.89 & 0.053 & $21 \%$ \\
\hline $\begin{array}{l}\text { Unprogressively motile } \\
\text { sperm cells after } 1 \text { hour (\%) }\end{array}$ & 5.73 & 3.29 & 7.69 & 3.86 & 0.029 & $34 \%$ \\
\hline $\begin{array}{l}\text { Motile spermatozoa after } \\
24 \text { hours (\%) }\end{array}$ & 21.9 & 17.3 & 16.4 & 15.1 & 0.178 & $-25 \%$ \\
\hline $\begin{array}{l}\text { Progressively motile } \\
\text { spermatozoa after } 24 \\
\text { hours (\%) }\end{array}$ & 6.52 & 8.89 & 6.33 & 7.61 & 0.925 & $-3 \%$ \\
\hline Normal morphology (\%) & 51.6 & 8.36 & 53.1 & 7.65 & 0.448 & $3 \%$ \\
\hline $\begin{array}{l}\text { Iron concentration in } \\
\text { seminal plasma }(\mu \mathrm{g} / \mathrm{dl})\end{array}$ & 1.80 & 0.84 & 6.44 & 3.89 & $<0.001$ & $258 \%$ \\
\hline
\end{tabular}

Table 2. Parameters related to oxidative stress intensity and antioxidant reserves in the seminal plasma and spermatozoa

\begin{tabular}{|c|c|c|c|c|c|c|}
\hline & \multicolumn{2}{|c|}{ Fe-L group } & \multicolumn{2}{|c|}{ Fe-H group } & \multirow{2}{*}{$p$ value } & \multirow{2}{*}{$\begin{array}{l}\text { relative } \\
\text { change }\end{array}$} \\
\hline & Mean & SD & Mean & SD & & \\
\hline $\begin{array}{l}\text { PSH concentration }(\mu \mathrm{mol} / \mathrm{g} \\
\text { protein) }\end{array}$ & 4.70 & 2.15 & 5.42 & 3.44 & 0.400 & $15 \%$ \\
\hline SOD activity (NU/mg protein) & 4.26 & 1.65 & 3.85 & 1.43 & 0.361 & $-10 \%$ \\
\hline $\begin{array}{l}\text { Mn-SOD activity (NU/mg } \\
\text { protein) }\end{array}$ & 0.56 & 0.37 & 0.62 & 0.56 & 0.643 & $11 \%$ \\
\hline $\begin{array}{l}\text { CuZn SOD activity ( } \mathrm{NU} / \mathrm{mg} \\
\text { protein) }\end{array}$ & 3.59 & 1.47 & 3.23 & 1.06 & 0.337 & $-10 \%$ \\
\hline $\begin{array}{l}\text { SOD activity (NU/100 } \mu \mathrm{l} \\
\text { packed spermatozoa) }\end{array}$ & 180 & 63.6 & 65 & 31 & $<0.001$ & $-64 \%$ \\
\hline $\begin{array}{l}\text { Mn-SOD activity (NU/100 } \mu \mathrm{l} \\
\text { packed spermatozoa) }\end{array}$ & 146 & 54.3 & 54 & 36.3 & 0.006 & $-63 \%$ \\
\hline $\begin{array}{l}\text { CuZn SOD activity (NU/100 } \mu \mathrm{l} \\
\text { packed spermatozoa) }\end{array}$ & 34.4 & 18.8 & 20.6 & 11.7 & 0.185 & $-40 \%$ \\
\hline $\begin{array}{l}\text { MDA concentration }(\mu \mathrm{mol} / \mathrm{dl} \\
\text { packed spermatozoa) }\end{array}$ & 0.55 & 0.21 & 0.57 & 0.19 & 0.878 & $2 \%$ \\
\hline MDA concentration $(\mu \mathrm{mol} / / \mathrm{I})$ & 2.74 & 1.25 & 2.04 & 0.76 & 0.010 & $-25 \%$ \\
\hline $\mathrm{TOS}(\mu \mathrm{mol} / / \mathrm{l})$ & 5.97 & 11.52 & 11.4 & 11.0 & 0.002 & $90 \%$ \\
\hline $\mathrm{TAC}(\mathrm{mmol} / \mathrm{l})$ & 1.33 & 0.27 & 1.25 & 0.27 & 0.406 & $-6 \%$ \\
\hline OSI (\%) & 0.50 & 0.95 & 0.94 & 0.83 & 0.002 & $89 \%$ \\
\hline
\end{tabular}

OSI were significantly higher in the Fe-H group, compared to the Fe-L group, by $90 \%$ and $89 \%$, respectively (Table 2 ).

Median of IL-1 $\beta$ was significantly higher in the Fe-H group, compared to the Fe-L group. Medians of IL-2, IL-5, and GM- 
Table 3. Concentrations of cytokines in the seminal plasma

\begin{tabular}{lccccc}
\hline & \multicolumn{2}{c}{ Fe-L group } & \multicolumn{2}{c}{ Fe-H group } & \multirow{2}{*}{ p value } \\
\cline { 2 - 5 } & median & IQR & median & IQR & \\
\hline IL-1 $\beta \mathrm{pg} / \mathrm{ml}$ & 0.62 & 0.77 & 2.0 & 7.1 & $\mathbf{0 . 0 0 8}$ \\
\hline $\mathrm{IL}-2 \mathrm{pg} / \mathrm{ml}$ & 0.96 & 5.33 & 0.05 & 0.30 & $\mathbf{0 . 0 3 9}$ \\
\hline $\mathrm{IL}-4 \mathrm{pg} / \mathrm{ml}$ & 0,11 & 0,34 & 0,09 & 0,03 & 0,181 \\
\hline $\mathrm{IL}-5 \mathrm{pg} / \mathrm{ml}$ & 45.6 & 194.3 & 33.0 & 49.7 & $\mathbf{0 . 0 3 8}$ \\
\hline $\mathrm{IL}-6 \mathrm{pg} / \mathrm{ml}$ & 8.7 & 16.0 & 11.1 & 11.1 & 0.436 \\
\hline $\mathrm{IL}-8 \mathrm{pg} / \mathrm{ml}$ & 171 & 228 & 143 & 129 & 0.193 \\
\hline IL-12 $(\mathrm{p} 70) \mathrm{pg} / \mathrm{ml}$ & 1.71 & 3.11 & 3.29 & 4.36 & 0.323 \\
\hline IFN- $\gamma \mathrm{pg} / \mathrm{ml}$ & 98,4 & 81,3 & 34,9 & 64,5 & 0,635 \\
\hline G-CSF pg/ml & 23.4 & 25 & 19.1 & 34 & 0.430 \\
\hline GM-CSF pg/ml & 220 & 48.9 & 188 & 53.4 & $\mathbf{0 . 0 4 5}$ \\
\hline
\end{tabular}

CSF were significantly lower in the Fe-H group, compared to the Fe-L group (Table 3).

Spearman correlation showed positive correlations between levels of Fe and TOS value $(R=0.48 ; p=0.003)$ and the percentage of unprogressively motile sperm cells after 1 hour $(\mathrm{R}=0.31 ; \mathrm{p}=0.012)$. Negative correlations between the levels of $\mathrm{Fe}$ and the percentage of progressively motile sperm cells after 1 hour $(\mathrm{R}=-0.34 ; \mathrm{p}=0.005)$, plasma MDA level $(\mathrm{R}=-0.36 ; \mathrm{p}=0.004)$, and activities of total SOD $(\mathrm{R}=-$ $0.63 ; \mathrm{p}=0.001)$ and $\mathrm{Mn}-\mathrm{SOD}(\mathrm{R}=-0.50 ; \mathrm{p}=0.03)$ in packed spermatozoa were also shown.

\section{DISCUSSION}

It has been shown that seminal Fe concentration may affect sperm motility [16]. The presented study shows that the Fe level in seminal plasma does not affect the percentage of motile sperm cells and non-motile sperm cells after 1 hour. However, results indicate that the Fe level may influence which types of motility are exhibited by sperm cells. Fe may be associated with a decreased percentage of progressively (rapid linear) motile sperm cells, an increased percentage of low progressively (slow linear), and unprogressively (non-linear) motile sperm cells. These changes in types of motility may contribute to the deterioration of sperm quality. Besides, a strong tendency was shown towards decreased sperm volume in the $\mathrm{Fe}-\mathrm{H}$ group. It is possible that higher Fe concentrations are secondary to a decreased secretory activity of the male accessory glands, accompanied by the unchanged total iron pool in the ejaculate.

However, the reverse association to the above is also worth discussing, although the results of other studies on this topic are inconclusive. Huang et al. [6] showed elevated Fe levels in a group of asthenospermic patients compared with the normospermic males. The authors of this study did not observe any significant correlations between Fe level and sperm count, motility, and MDA level in the normospermic subjects. However, in a group of subjects with combined sperm abnormalities, Fe level correlated positively with sperm count, but not motility and MDA. In a study by Aydemir et al. [17], seminal plasma Fe level was shown to be increased in subfertile males, compared to fertile ones. A positive correlation between Fe level and sperm motility was also shown in this study. Marzec-
Wróblewska et al. [1] showed no significant differences between Fe levels in normozoospermic males and males with pathological spermiogram; however, Fe level in seminal plasma correlated negatively with normal sperm morphology. The above-mentioned results are not in concordance with those of Skandhan et al. [16] who found decreased Fe levels in males diagnosed with oligoasthenozoospermia and asthenozoospermia. The authors of this study concluded that an optimum physiological range for sperm iron level is necessary, and any alteration in its concentration on either side of this range may result in adverse effects on motility. In the other above-mentioned studies, the role of Fe as a catalyst of oxidative stress and lipid peroxidation is emphasized and supposed to be associated negatively with semen quality $[1,17]$.

In the presented study, a highly-elevated level of TOS value in the $\mathrm{Fe}-\mathrm{H}$ group and a positive correlation between the Fe level and TOS value was consistently found. Because a decreased level of MDA and a negative correlation between the levels of Fe and MDA were found, this elevation may be due to the increased level of other oxidative damage products, e.g. hydroperoxides of lipids. The TOS value elevation in the $\mathrm{Fe}-\mathrm{H}$ group was not accompanied by a decreased value of TAC and level of thiol groups. Therefore, it is possible that the proposed $\mathrm{Fe}$-induced oxidative stress triggers a compensatory antioxidant defence mechanism, resulting in decrease in the MDA level. A part of this antioxidant defence mechanism is SOD which catalyzes the reduction of superoxide anions to hydrogen peroxide. SOD has been shown to play a critical role in the defence of cells against the toxic effects of oxygen radicals, and maintaining sperm viability and motility. Besides, a positive correlation between seminal plasma activity of SOD and seminal plasma level of Fe was previously found. This elevation of SOD activity was proposed to be a defensive response to the Fe-induced oxidative stress [1]. In the presented study, no associations were found between the Fe level and SOD activity in seminal plasma. Surprisingly, decreased activities were found of total SOD and Mn-SOD in packed spermatozoa obtained from subjects in the $\mathrm{Fe}-\mathrm{H}$ group. Negative correlations were also found between $\mathrm{Fe}$ levels and SOD activities. This data suggests that Fe action may depend on a particular sperm compartment and should be investigated in each compartment separately.

The intensity of oxidative stress in semen and semen quality was shown to be affected by pro-inflammatory cytokines, such as IL-1 $\beta$, IL-6, IL- 8 , and IL-12 $[18,19]$. In the presented study, median of IL- $1 \beta$ levels in the $\mathrm{Fe}-\mathrm{H}$ group was higher than in the Fe-L group. This observation suggests that Fe may also deteriorate semen quality through elevation of the IL-1 $\beta$ level. Besides, a lower median of IL- 2 and IL-5 levels was observed in the Fe-H group, compared to the Fe-L group. The role of these cytokines in sperm is poorly understood. It is known that IL-2 activates Th type 1 (Th1) response, while IL-5 promotes humoral immunity activating Th type 2 (Th2) response [20]. IL-5 receptors are expressed in the germ line of human testis and ejaculated sperm, which indicate that this cytokine may play a role in the physiology of the human testis [21]. Other cytokines related to the Th1 or Th2 responses did not differ between the examined groups. Therefore, it is not possible to suppose that Fe is able to promote cellular or cytotoxic immunity response.

Colony stimulating factors (CSFs) have been found to enable fertilization. Seminal fluid from infertile men 
is characterized by lower levels of G-CSF and GM-CSF, compared with seminal fluid from normal fertile donors [22]. Besides, GM-CSF is able to enhance several parameters of sperm motility in the presence of glucose or fructose in vitro [23]. The relationship between the level of Fe and median of GM-CSF, which was shown in the presented study, may serve as one more explanation for Fe-induced modifications of sperm motility parameters which were simultaneously observed.

\section{CONCLUSIONS}

In the examined fertile males, Fe negatively affected some parameters characterizing sperm motility and an elevated TOS value, which reflects oxidative stress intensity. Fe also modulated the levels of several cytokines. The influence of Fe on of IL-1 $\beta$ and GM-CSF levels may have contributed to the deterioration of semen motility.

$\mathrm{Fe}$ is indispensable for normal cell function, and the threshold of its toxicity in human semen seems to be relatively low.

\section{REFERENCES}

1. Marzec-Wróblewska U, Kamiński P, Lakota P, Szymański M, Wasilow K, Ludwikowski G, Kuligowska-Prusińska M, Odrowąż-Sypniewska G, Stuczyński T, Michałkiewicz J. Zinc and iron concentration and SOD activity in human semen and seminal plasma. J Biol Trace Elem Res. 2011; 143(1): 167-177.

2. Morales C, Sylvester SR, Griswold MD. Transport of iron and transferrin synthesis by the seminiferous epithelium of the rat in vivo. Biol Reprod. 1987; 37(4): 995-1005.

3. Metzendorf C, Lind MI. Drosophila mitoferrin is essential for male fertility: evidence for a role of mitochondrial iron metabolism during spermatogenesis. BMC Dev Biol. 2010; 10: 68. doi: 10.1186/1471-213X10-68.

4. Perera D, Pizzey A, Campbell A, Katz M, Poter J, Petrou M, Irvine DS, Chatterjee R Sperm DNA damage in potentially fertile homozygous $\beta$-thalassaemiapatients with iron overload. Hum Reprod. 2002; 17(7): 1820-1825.

5. Ayinde OC, Ogunnowo S, Ogedegbe RA. Influence of Vitamin C and Vitamin E on testicular zinc content and testicular toxicity in lead exposed albino rats. BMC Pharmacol Toxicol. 2012; 14: 13-17.
6. Huang YL, Tseng WC, Lin TH. In vitro effects of metal ions $\left(\mathrm{Fe}^{2}+\right.$, $\mathrm{Mn}^{2+}, \mathrm{Pb}^{2+}$ ) on sperm motility and lipid peroxidation in human semen. J Toxicol Environ Health 2001; 23(62): 259-267.

7. Sharma RK, Pasqualotto FF, Nelson DR, Thomas AJ Jr, Agarwal A The reactive oxygen species-total antioxidant capacity score is a new measure of oxidative stress to predict male infertility. Hum Reprod. 1999; 14(11): 2801-2807.

8. Potts JM, Pasqualotto FF. Seminal oxidative stress in patients with chronic prostatitis. Andrologia. 2003; 35(5): 304-308.

9. Seshadri S, Bates M, Vince G, Jones DI. The role of cytokine expression in different subgroups of subfertile men. Am J Reprod Immunol. 2009; 62(5): 275-282.

10. WHO. Laboratory manual for the examination of human semen V ed. Cambridge University Press 2010.

11. Koster JF, Biemond P, Swaak AJ. Intracellular and extracellular sulphydryl levels in rheumatoid arthritis. Ann Rheum Dis. 1986; 45: 44-46.

12. Oyanagui Y. Reevaluation of assay methods and establishment of kit for superoxide dismutase activity. Anal Biochem 1984; 142: 290-296.

13. Ohkawa H, Ohishi N, Yagi K. Assay for lipid peroxides in animal tissues by thiobarbituric acid reaction. Anal Biochem. 1979; 95: 351-358.

14. Erel O. A new automated colorimetric method for measuring total oxidant status. Clin Bioch. 2005; 38: 1103-1111.

15. Erel O. A novel automated direct measurement method for total antioxidant capacity using a new generation, more stable ABTS radical cation. Clin Bioch. 2004; 37: 277-285.

16. Skandhan KP, Mazumdar BN, Sumangala B. Study into the iron content of seminal plasma in normal and infertile subjects. Urologia. 2012; 79(1): 54-57.

17. Aydemir B, Kiziler AR, Onaran I, Alici B, Ozkara H, Akyolcu MC. Impact of $\mathrm{Cu}$ and $\mathrm{Fe}$ concentrations on oxidative damage in male infertility. Biol Trace Elem Res. 2006; 112(3): 193-203.

18. Fraczek M, Sanocka D, Kamieniczna M, Kurpisz M. Proinflammatory cytokines as an intermediate factor enhancing lipid sperm membrane peroxidation in in vitro conditions. J Androl. 2008; 29(1): 85-92.

19. Martínez P, Proverbio F, Camejo MI. Sperm lipid peroxidation and pro-inflammatory cytokines. Asian J Androl. 2007; 9(1): 102-107.

20. Shen X, Lee K, König R. Effects of heavy metal ions on resting and antigen-activated CD4(+) T cells. Toxicology. 2001; 169(1): 67-80.

21. Politch JA, Tucker L, Bowman FP, Anderson DJ. Concentrations and significance of cytokines and other immunologic factors in semen of healthy fertile men. Hum Reprod. 2007; 22(11): 2928-2935.

22. Ota K, Jaiswal MK, Ramu S, Jeyendran R, Kwak-Kim J, Gilman-Sachs A, Beaman KD. Expression of a2 vacuolar ATPase in spermatozoa is associated with semen quality and chemokine-cytokine profiles in infertile men. PLoS One. 2013; 30;8(7):e70470. doi: 10.1371/journal. pone.0070470. Print 2013.

23. Rodríguez-Gil JE, Silvers G, Flores E, Jesús Palomo M, Ramírez A, Montserrat Rivera M, et al. Expression of the GM-CSF receptor in ovine spermatozoa: GM-CSF effect on sperm viability and motility of sperm subpopulations after the freezing-thawing process. Theriogenology. 2007; 67(8): 1359-1370. 